and choroid, due to the action of micro-organisms or their toxins, there is an overgrowth of the pigment epithelium and a migration of masses of these cells of various sizes into the retina. But the outgrowth of a pigment process of considerable length, which carries before it the layers of the retina into the middle of that structure, seems new. Furthermore, in this case, there is no evidence of an infection with micro-organisms, and the choroid is unaffected, except for a slight degree of colloid degeneration of the membrane of Bruch-of the inner or cuticular lamella of the lamina vitrea. The fact that, in retinal detachment, the separation takes place between the retina and the pigment epithelium layer, would seem to discount the remote possibility that this microscopic appearance resulted from an extreme dimpling of the retina with its pigment epithelium from without, on restoration to its position against the choroid.

Summary. Macroscopic examination reveals no trace of retinal detachment in either eye. Microscopic examination discloses no retinal detachment in either, but shows a slight amount of exudate in Henle's layer in the left eye, and also a peculiar localized pigment proliferation in the same locality of the retina.

\title{
A CASE OF TUBERCULOUS IRIDOCYCLITIS AND PARENCHYMATOUS KERATITIS OF THE LEFT EYE, ASSOCIATED WITH TUBERCULOSIS OF THE CON- JUNCTIVA OF THE RIGHT EYE, AND TUBERCULOUS LYMPHADENITIS*
}

\author{
BY \\ Humphrey Neame, F.R.C.S. \\ LONDON
}

Herbert Dyke, aged 19 years, came under the care of Mr. E. Treacher Collins at the Royal London Ophthalmic Hospital in June, 1921. The patient's occupation was that of a boot and shoe stock-keeper. He was sent to this hospital from the Victoria Park Hospital on account of loss of sight in the left eye.

His history is as follows : Dyke stated that his general health depreciated during and after a fourteen days' holiday at Southend in August, 1920. He had cycled there from London, and on his arrival felt very tired. Throughout his stay there he felt unable to exert himself. After his return to work in London, one day while stooping down he felt something " go " in his left eye, and then saw "black spots and a flash" with this eye. From that

"Shown at the meeting of the Ophthalmic Section of the Royal Society of dicine, on Friday, November 11, 1921. 
date the sight of the left eye steadily diminished, until in six weeks he could only see his hand moved in front of it. About September, 1920, he noticed the appearance of lumps in the right and left sides of his neck. During this time he attended the Out-Patient Department of the Metropolitan Hospital. About six months laterMarch, 1921-he began to cough and he was troubled by the right eye which watered. In May, 1921, he noticed that he was becoming thinner. Dyke then went to the City of London Hospital for Diseases of the Chest, Victoria Park, where he was detained as an in-patient for five weeks under the care of Dr. O. K. Williamson.

By the kindness of Dr. C. Kingston, Registrar of the Victoria Park Hospital, information was received by the writer that no signs of tuberculosis had been found in the chest, that the sputum revealed the presence of no tubercle bacilli, and that a diagnosis of Hodgkin's disease had been made in connection with the glandular enlargement. Dyke stated that his weight, which had been taken weekly while he was in the hospital, had remained fairly constant, about 8 st. $5 \mathrm{lbs}$. in his ordinary indoor clothes. He further stated that he had never suffered from a prolonged cough until August, 1920. He started coughing shortly after his return from the Southend holiday, and had been troubled with a cough to some extent every day since then. He had no pain in the chest, but slight shortness of breath on exertion since the onset of the cough. This shortness of breath had improved latterly. He had coughed up sputum while he had been in Victoria Park Hospital, but less on his discharge from hospital, and very little since then.

\section{Further Ophthalmic History}

June, 1921. Dyke was admitted to the Royal London Ophthalmic Hospital.

There was then marked lymph gland enlargement in the anterior and posterior and suboccipital triangles of the neck on each side. The glands were smooth, firm, discrete and fairly mobile, and not adherent to the skin.

The right eye showed conjunctival injection, palpebral and ocular, and some enlarged follicles in the conjunctiva of the lower lid and to a slight extent at the inner and outer angles of the fornix of the upper lid. There were also on the lower lid several conjunctival nodules, of translucent gelatinous appearance and grey-white colour, with particularly marked injection of the conjunctiva immediately around. Single nodules were about $1 \mathrm{~mm}$. in diameter. Some of the nodules were of irregular shape as if they were formed by confluence of two or three nodules. There were in the conjunctiva of the upper lid three separate very small nodules of similar appearance. 
The cornea was clear and bright, the iris of healthy appearance and the pupil circular and active. The tension was normal.

The vision of the right eye, while under the effect of a mydriatic, with correction-4.0 D. sphere, -2.0 D. cyl. axis 20 degrees, was $6 / 12$ four letters, without correction $6 / 60$. The media were clear except for two small white dots near the centre of the anterior lens capsule, and the fundus was healthy.

The left eye showed conjunctival and ciliary injection. The cornea was vascularized and showed a general haze, superficial and deep, sufficiently dense to obscure details of all structures behind it. The lower part of the cornea was to a slight degree staphylomatous. Vision was perception of light only, and the tension was reduced.

The left eye was excised, fixed in formalin and bisected vertically between disc and macula and through the corneal staphyloma.

\section{Pathology}

Macroscopic examination. Left eyeball. (Formalin fixation, vertical antero-posterior bisection.) The cornea on section appears clear except for the lower part which is staphylomatous. Here the opaque cornea is continuous with a white mass behind it. The anterior chamber is almost obliterated, filled up with a homogeneous white mass which is continuous with remnants of infiltrated iris and ciliary body. The iris and ciliary body are only identified by the pigment tissue in their posterior surfaces; they are blended with the white mass. The lens is displaced slightly downwards. There is a semi-transparent blue-grey membrane behind the lens and ciliary body in the anterior part of the vitreous chamber. The retina shows folds here and there, and a small detachment anteriorly and below, with greenish subretinal exudate of gelatinous appearance and firm consistency. The choroid is in situ.

Microscopic examination. Celloidin embedding. Vertical section, sagittal plane. Sections were stained with Ehrlich's acid haematoxylin and eosin, with Weigert's iron-haematoxylin and van Gieson's picric acid fuchsin, and with Ziehl-Neelsen's carbol fuchsin.

Cornea. There is oedema and round-cell infiltration of the deeper layers of the epithelium. Bowman's membrane is intact. In the periphery there is a thin zone of cellular infiltration deep to Bowman's membrane. The substantia propria is vascularized, and infiltràted, mainly with small round cells, and chiefly in its deeper layers (see Fig. 2).

Descemet's membrane disappears abruptly at its upper and lower extremities in dense cell infiltration in the neighbourhood of the site of the canal of Schlemm, which is not visible. The central 
portion of Descemet's membrane is seen in some sections to be deeply convoluted, and to have suffered some longitudinal splitting into fibrils, without actual rupture. In other sections it cannot

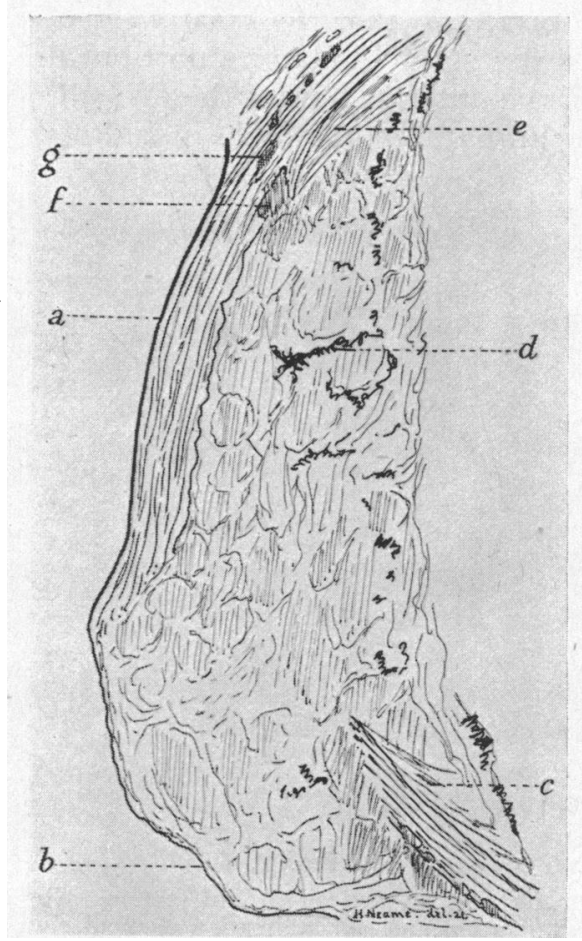

FIG. 1.

Anterior part of eye, vertical sagittal section. $\times 5$. Shows the extensive inflammatory new-formation, which fills the anterior chamber, obliterates almost all trace of the iris and ciliary body, and perforates the limbus inferior. This section is made well to one side of the middle vertical plane, so that many ciliary processes are cut across.

(a) Corneal epithelium.

(b) Conjunctival epithelium covering the staphyloma.

(c) Remnants of ciliary muscle internal to the sclera immediately posterior to the perforation.

(d) Remnant of ciliary pigment epithelium.

(e) Ciliary muscle in the upper part of globe.

(f) Canal of Schlemm filled with cellular exudate, or obliterated by pressure of the exudate in the lymphatics around the cana!.

(g) Tributary of anterior ciliary vessel, also filled with, or obliterated by cellular exudate. No endothelium is seen in these spaces The cellular exudate has the same structure as that of the main mass.

be traced continuously from the upper to the lower part of the cornea, and in these there is a more extensive replacement of substantia propria by inflammatory cell infiltration.

The limbus or just posterior to it-as far as can be judged, 
opposite to the angle of the anterior chamber-is perforated above, in a narrow zone, by a mass of round and spindle-shaped cells and new fibrous tissue. Below there is a similar condition, except that the zone of penetration is wider, that there is a thin covering of substantia propria, and that the area affected is prominent and corresponds with the staphylomatous portion already mentioned. A thin portion of the anterior half of the eyeball was embedded by Jordan's photoxylin-paraffin method, including the most promi-

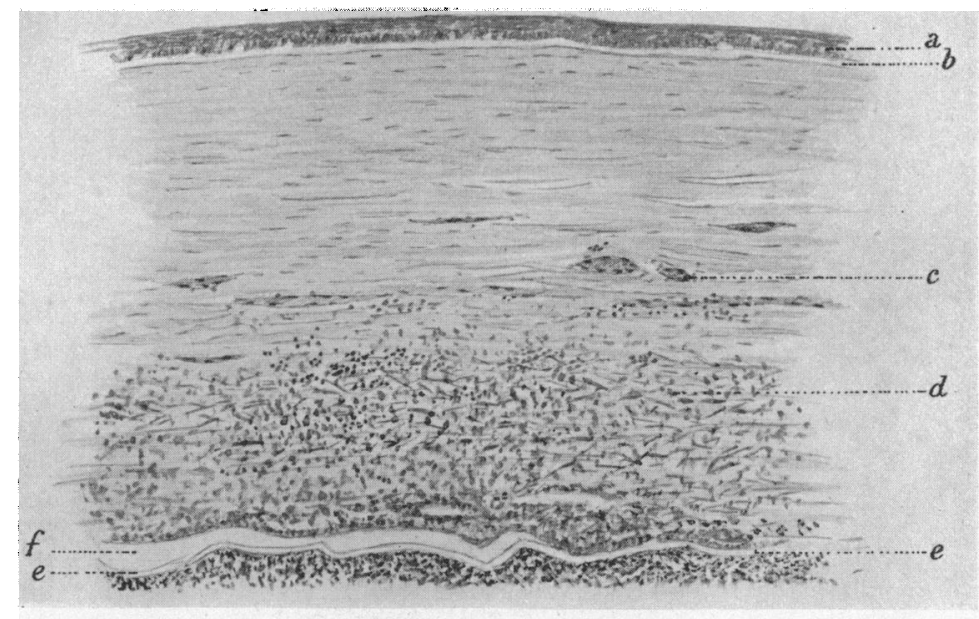

Fig. 2.

Cornea, near its centre, vertical sagittal section. Shows tuberculous keratitis, vascularization and cell infiltration particularly of deeper part, and corrugation of Descemet's membrane.

(a) Epithelium.

(b) Bowman's membrane.

(c) Vessels in substantia propria.

(d) Cellular infiltration.

(e) Descemet's membrane.

(f) Artefact, separation of Descemet's membrane. Deep to Descemet's membrane is the mass of cell and fibrous new-formation in the anterior chamber.

nent portion of the staphyloma. In sections of this part (Fig. 1) it is evident that a complete perforation has taken place at the inferior portion of the limbus, and that the direction of this perforation has been similar to that of the usual large rupture from indirect violence (1), namely, obliquely outwards from the canal of Schlemm, along the plane of the anterior ciliary veins as they pass outwards through the sclera. The free ends of the ruptured coats of the globe show this obliquity both anterior and posterior -or superior and inferior-to the perforation. These ends are separated at this site by a space of about $3 \mathrm{~mm}$. which is occupied by fibro-cellular inflammatory mass. The free end of sclera 


\section{Tuberculous Iridocyclitis and Parenchymatous Keratitis 209}

inferior to the perforation is overlapped on its outer surface by the same mass for an extent of fully $2 \mathrm{~mm}$. The granulomatous tissue is covered on its outer surface by stretched corneal and conjunctival epithelium and a small amount of vascular sub-conjunctival connective tissue. Bowman's membrane is lost in cellular infiltration before it reaches the protruding mass.

The anterior chamber is abolished-in the sections examined,

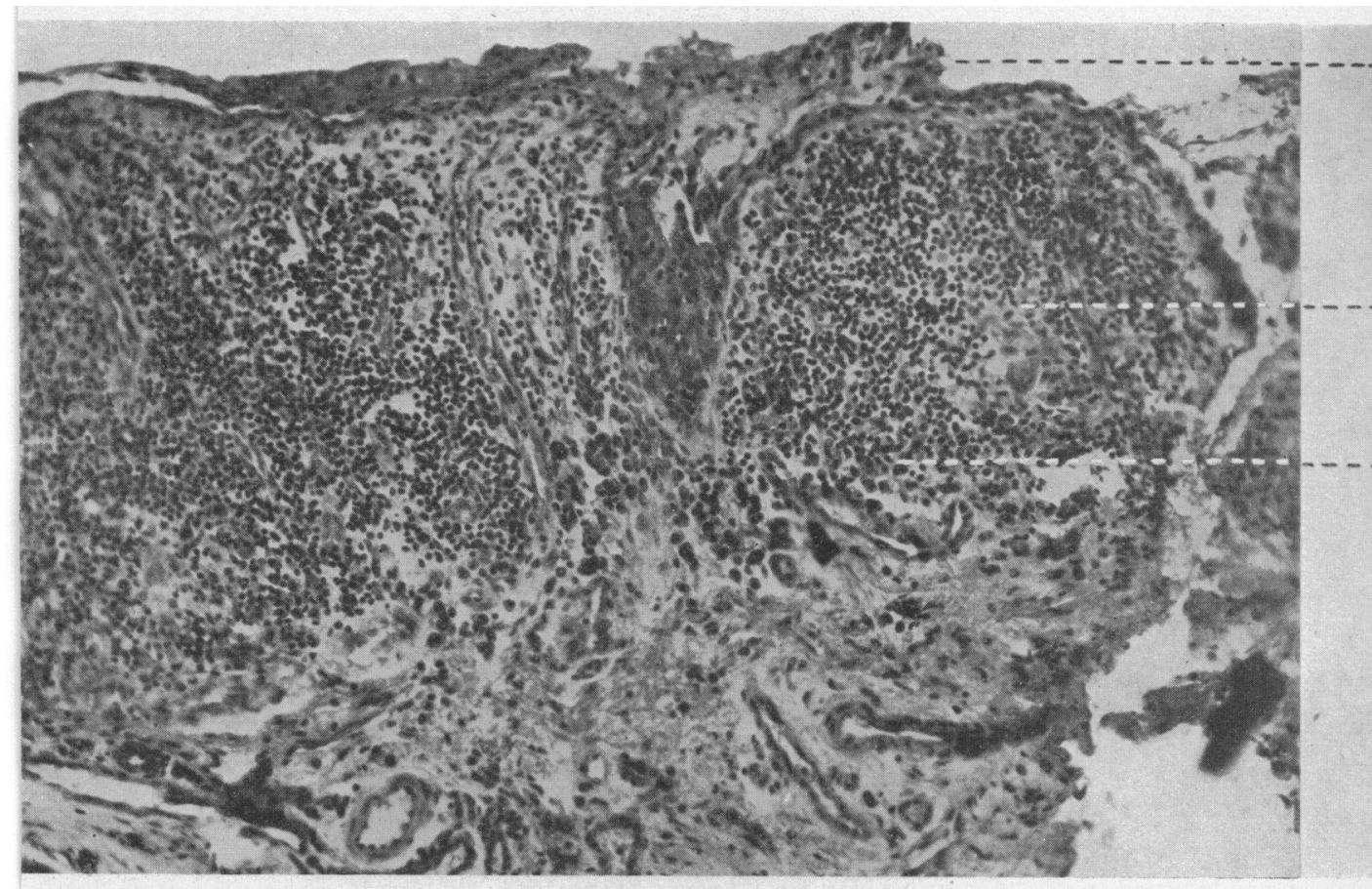

FIG. 3.

Conjunctiva of lozerer lid (Micro-photo). $\times 150$ Shows two adjacent nodules.

(a) Stretched and atrophic epithelium

(b) Centre of one nodule containing a Langhans' giant cell and epithelioid cells.

(c) Surrounding zone of round-cell infiltration, outside of which is fibrous tissue.

which are taken from the vertical central portion of the corneaexcept for a narrow cleft seen in a few sections, anterior to the lens and bounded in front by a mass of inflammatory tissue containing traces of iris pigment. The chamber is filled with a mass composed of confluent nodules of small round cells, epithelioid cells, spindle-cells and fibrous tissue, and a few giant-cells of Langhans' type with homogeneous cell protoplasm and peripheral nuclei. 
The iris and ciliary body are largely destroyed, and replaced by a similar inflammatory formation which contains streaks and patches of pigment mainly in its posterior region. This inflammatory infiltration extends back to about half-way along the pars plana, and has destroyed by infiltration the epithelium of nearly the anterior half of the pars plana.

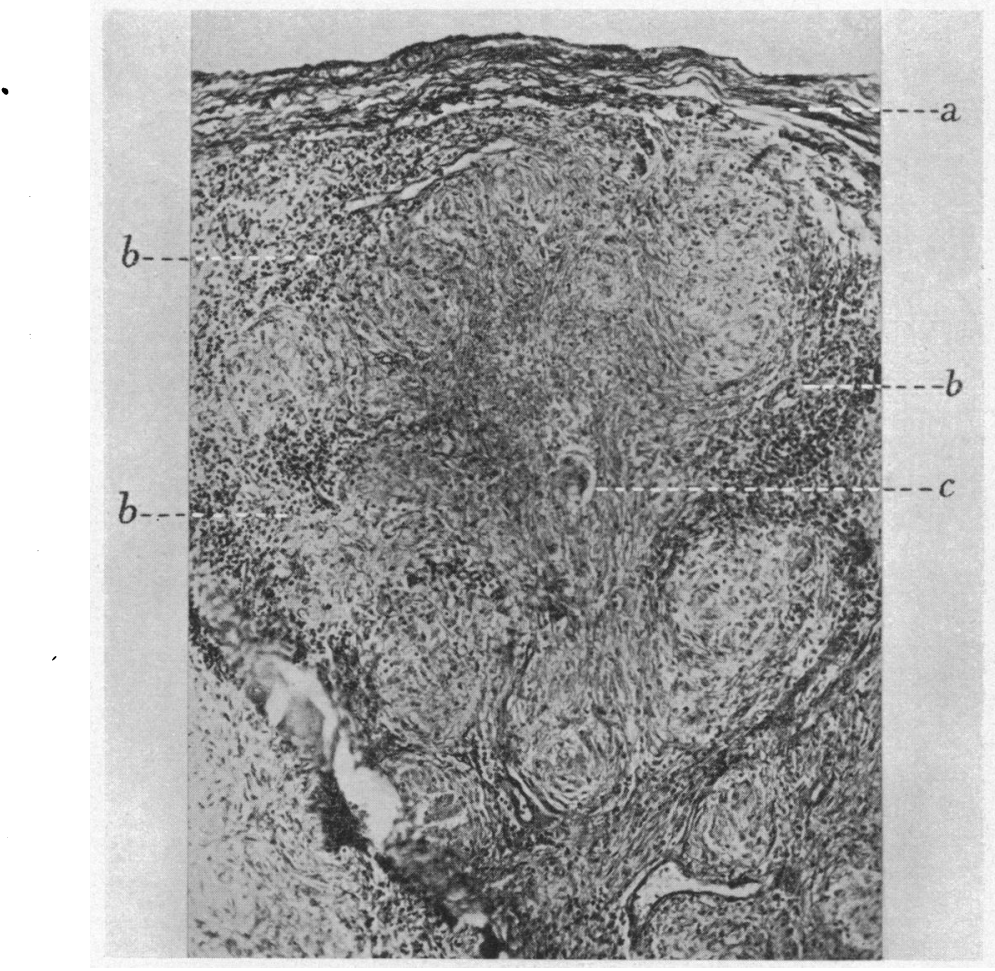

FIG 4.

Lymphatic. gland (Micro-photo.). $\times 100$, from posterior auricular region. Stain, Weigert iron haematoxylin and van Gieson picric acid fuchsin stain. Shows :-

(a) Fibrous capsule of gland.

(b) Remnants of lymph-cell formation.

(c) Large ill-formed giant-cell.

The main structure is very rich in collagen fibre stained red with van

Gieson stain. This can be seen to be arranged in a nodular formation.

The Lens shows a well-mạked anterior capsular cataract, formed of very long spindle shaped cells. Between and around the fibres of the suspensory ligament of the lens is an extension of the inflammatory mass, and in the vitreous immedately posterior to this are groups of small round cells.

The retina, choroid and optic nerve are normal. 
On July 21, 1921, a fragment of conjunctiva was removed from the right lower lid in its outer part, including several of the greywhite translucent nodules already described. In the interval since the removal of the left eye the nodules in the conjunctiva of the right lower lid had slightly increased in size. Half of the tissue, pinned out on card, was fixed in Zenker's fluid for eight hours, treated in the ordinary manner and embedded in paraffin. The other half was introduced into the subcutaneous tissue of a guinea pig.

Sections of the conjunctiva were stained with Ehrlich's haematoxylin and eosin, with van Gieson's picric-acid fuchsin and Weigert's iron haematoxylin, with Pappenheim's stain, and with Ziehl-Neelsen's carbol-fuchsin. The microscopic appearance of the sections was that of a granulomatous condition of the conjunctiva with a moderate amount of fibrous tissue formation. The sections stained for tubercle bacilli failed to disclose any organisms. An isolated nodule removed at the same time from the upper lid was teased out and stained by the same method by Dr. S. H. Browning with a similar negative result.

The guinea-pig inoculation of conjunctiva was performed by Dr. P. Fildes at the London Hospital. This also gave a negative result.

On September 29, 1921, with Mr. Treacher Collins's permission, a further piece of conjunctiva was removed from the right lower lid, and two small discrete lymph glands were excised from the subcutaneous tissue behind the right ear. One lymph gland was used for a further inoculation test on a guinea-pig by Dr. Fildes. The weight of the guinea-pig was as follows :--

$\begin{array}{rllll}29 / 9 / 21 & \ldots & \ldots & 360 & \text { Grams. } \\ 7 / 10 / 21 & \ldots & \ldots & 370 & , \\ 13 / 10 / 21 & \ldots & \ldots & 440 & , \\ 22 / 10 / 21 & \ldots & \ldots & 450 & , \\ 10 / 11 / 21 & \ldots & \ldots & 440 & ,\end{array}$

On November 10 there was a firm swelling in the subcutaneous tissue at the site of inoculation, adherent to the abdominal wall, with the skin movable over it. The animal was killed and the swelling was opened.

Dr. Fildes' report follows :-

“ London Hospital, Bacteriological Department, November 10, 1921.

Guinea-pig 360 grams. subcutaneous inoculation $29 / 9 / 21$, with a whole lymphatic gland $(8 \mathrm{~mm}$. diam.) ground up in sand.

$10 / 11 / 21$ weight 440 grams. Killed. 
Small local subcutaneous abscess, containing dry necrotic pus.

No tubercle bacilli found. No tuberculosis elsewhere.",

The other lymph gland and the conjunctiva were fixed in Zenker's fluid, treated as usual and embedded in paraffin.

Histology of the conjunctiva and lymph gland. The serial sections of part of this specimen of conjunctiva were stained with Ehrlich's haematoxylin and eosin, or with Weigert's iron haematoxylin and van Gieson's picric-acid fuchsin stains. They show in section several distinct nodules up to 0.5 millimetre in their longest dimensions. (See Fig. 3.) These nodules are subdivided into smaller nodules of cells by slender collagen fibres-as shown by van Gieson's stain-and the smallest of these subsidiary nodules measures $\tau_{0} \mu$ in diameter. There are seen in some of the sections, well formed nodules bounded by fibrous tissue, composed mainly of small round cells, with a central zone of epithelioid cells and a giant cell among them, and with a few of the plasma type of cell.

Sections of the lymphatic gland were stained in a manner similar to that of the conjunctiva, and in addition with Pappenheim's pyronin methyl green stain. The sections show the gland with a dense fibrous tissue covering. This covering in places sends many collagen fibres into the gland itself, to blend with an abundance of collagen fibres in the stroma. The normal lymphatic gland structure has disappeared. There are but a few small islands of lymphocytes here and there. The bulk of the gland is formed of rounded nodules, of size similar to that of the conjunctival nodules, but composed of epithelioid cells and fibroblasts (see Fig. 4). There are a few scattered lymphocytes, and in some of the nodules in section only two or three of these cells are to be seen. The most marked feature in the sections stained by van Gieson's method, is the fibrous tissue formation. Around and between the nodules there is an abundance of collagen fibres, and into most of the nodules pass numbers of slender fibres of this variety. No areas of necrosis are to be seen. In the presence of such a large amount of fibrous tissue in the gland, it seemed certain that the tubercle bacillus would not be found, and no sections were therefore stained with carbol fuchsin.

\section{Conclusions}

Lymph glands. It is possible that there are two separate pathological conditions present at one and the same time. As far as the lymphatic gland enlargement is concerned, the diagnosis lies between tuberculous lymphadenitis and Hodgkin's disease-or lymphadenoma.

Although the result of the inoculation of the tissue of one complete but small lymphatic gland into a guinea-pig was negative, 
this does not exclude the presence of tuberculosis. Nor does the absence of signs of tuberculosis in the lungs and of tubercle bacillus in the sputum lead necessarily in the same direction. This evidence is entirely negative, and merely a lack of support to the diagnosis of tuberculous lymphadenitis. An explanation of this failure is found in the histological examination of the lymph gland. The extreme fibrosis evident in sections stained by van Gieson's method, and the replacement of almost all lymphatic tissue thereby, indicates that the tuberculosis, if present, is of a very chronic form and vigorously resisted by the tissues. This readily accounts for the fact that tubercle bacilli were not found. In very chronic forms of tuberculosis, as lupus vulgaris, the search for the tubercle bacillus in the lesion entails much labour and is not often successful. Further, in such conditions, the result of guinea-pig inoculation is not invariably the production of tuberculosis in the animal. Such fibrosis of lymphatic glands is found in advanced stages of Hodgkin's disease, as well as in very chronic tuberculosis. But the nodular arrangement of the fibrous tissue throughout the gland, as in this case, supports the diagnosis of tuberculosis.

To summarize the evidence bearing upon the diagnosis of the lymph gland condition, we have-

(1.) In support of that of Hodgkin's disease.

(a) Absence of necrosis or caseation, although even in Hodgkin's disease this sometimes occurs ${ }^{(2)}$.

(b) Marked fibrosis in the gland.

(c) Negative bacteriological results to section examination and inoculation.

(2.) That these three facts $(a),(b)$ and $(c)$ are not evidence against the presence of a very chronic tuberculosis.

(3.) In favour of tuberculosis-(a) The nodular histological structure within the lymph gland, and $(b)$ the absence of eosinophile cells.

Iridocyclitis. (a). The presence of an extensive granulomatous formation of slightly nodular structure, in anterior chamber, iris and ciliary body, $(b)$ the erosion and perforation of Descemet's membrane and of the whole thickness of the cornea with the production of parenchymatous keratitis, are sufficient evidence, taken in conjunction with the history and clinical condition, to warrant the diagnosis of tuberculous iridocyclitis and keratitis.

Conjunctivitis. Although the histological structure of the nodules described bears some resemblance to that of early trachoma follicles and also of ophthalmia nodosa ${ }^{(3)}$ or pseudo-tuberculosis ${ }^{(4)}$, the presence of the nodules in this case on the palpebral conjunctiva only, and the absence of caterpillar hairs in any of the histological sections opposes the latter diagnosis, and the absence of 
other typical signs of trachoma, and the presence of tuberculosis elsewhere, are sufficient grounds for the diagnosis of the miliary type of tuberculosis of the conjunctiva.

It seems justifiable, therefore, to conclude that all the pathological conditions are due to one cause, namely, tuberculosis.

\section{REFERENCES}

1. Fuchs, E.-"Regarding some unusual varieties of rupture of the sclera." Ophthalmology. Vol. X, 1914, p. 46.

2. Webster, L. T.-" Lymphosarcoma. Lymphatic Leukaemia. Leucosarcoma. Hodgkin's Disease." Johns Hopkins Hospital Reports, Vol. XX, Part iii, p. 308.

3. Lawford, J. B.- "Ophthalmia Nodosa."-Trans. Ophth. Soc., Vol. XV, 1895, p. 210.

4. Stargardt, D. K.- "Ueber Pseudo Tuberculose des Auges." Arch.f. Ophthal., Vol. LV. 1903, p. 469.

\section{CLINICAL NOTES}

\section{THE DIAGNOSIS OF SUB.RETINAL TUMOUR}

BY

A. J. Ballantyne, M.D.

GLASGOW

A CASE of detachment of the retina in which there is reason to suspect the presence of a sub-retinal tumour may give one a good deal of anxiety, and one does not feel justified in proposing excision of the affected eye before having employed every method which will help to make the presence of a neoplasm reasonably certain. I have sometimes been disappointed with the results. of transillumination, and in two recent cases I have tested the character of the detachment by noting the effects of puncturing it under direct ophthalmoscopic examination. I mentioned the method at the November meeting of the Scottish Ophthalmological Club, and as it seemed to be new to the members I bring it forward in the hope that it may be helpful to others.

The case in which I employed it most recently was that of a woman, 46 years of age, presenting a prominent detachment of the retina in the lower nasal part of the fundus, highly suggestive of a sub-retinal neoplasm. Tension was normal, and there had been no pain or redness of the eye. Transillumination gave a doubtful result. A scleral puncture at the supposed site of the tumour gave exit to a small quantity of yellowish glairy fluid, but made no appreciable change in the prominence of the detachment. A few days later, under a local anaesthetic, a discission needle was inserted through the sclerotic in the upper and outer segment of the globe, passed across the vitreous, and, under direct observa- 